\title{
FLOW SEPARATION IN OUT-OF-ROUND NOZZLES, A NUMERICAL AND EXPERIMENTAL STUDY
}

\author{
C. Génin ${ }^{1}$, R. Stark ${ }^{1}$, and S. Jack ${ }^{2}$ \\ ${ }^{1}$ German Aerospace Center (DLR), Institute for Space Propulsion \\ Lampoldshausen 74239, Germany \\ ${ }^{2}$ German Aerospace Center (DLR) \\ Institute of Aerodynamics and Flow Technology \\ Braunschweig 38108, Germany
}

\begin{abstract}
The start-up and shutdown transient phases in rocket engine nozzles generate high side loads. The internal and external flow fluctuations can excite the eigenmodes of the nozzle structure. The flow separation in out-of-round nozzle intensifies the initial structure deformation leading in the worst case to the collapse of the nozzle. In the framework of the DLR internal cooperation program ProTAU, the ovalization of nozzles is being investigated from both the numerical and the experimental point of view. Three nozzle geometries have been designed and deformed applying various methods. The flow behavior in the initially axisymmetric and the ovalized contours has been numerically investigated. The most promising contours will be manufactured as permanently ovalized nozzle models and tested under cold flow conditions.
\end{abstract}

\section{INTRODUCTION}

The flow inside a rocket nozzle interacts with its structure. As the flow expands under ambient pressure conditions, the nozzle can be divided into two zones: in the first one, the inner wall pressure exceeds while in the second one, it is lower than the ambient pressure (Fig. 1a). Due to the local pressure differences, forces are applied onto the nozzle structure. If the nozzle is perfectly round and the flow is symmetrically distributed, the radial forces will compensate each other. This ideal circumferential distribution does not take place in reality, and so, side loads act, bending the nozzle and loading the engine, its actuators, and the rocket structure.

Two flow conditions must be differentiated depending on the nozzle pressure ratio (NPR, ratio of total over ambient pressure). For high NPR values, the

This is an Open Access article distributed under the terms of the Creative Commons Attribution License 4.0, which permits unrestricted use, distribution, and reproduction in any medium, provided the original work is properly cited. 


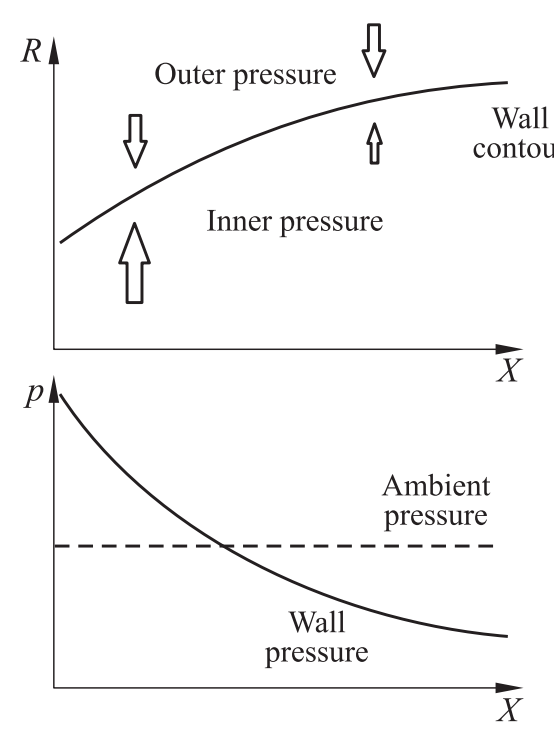

(a)

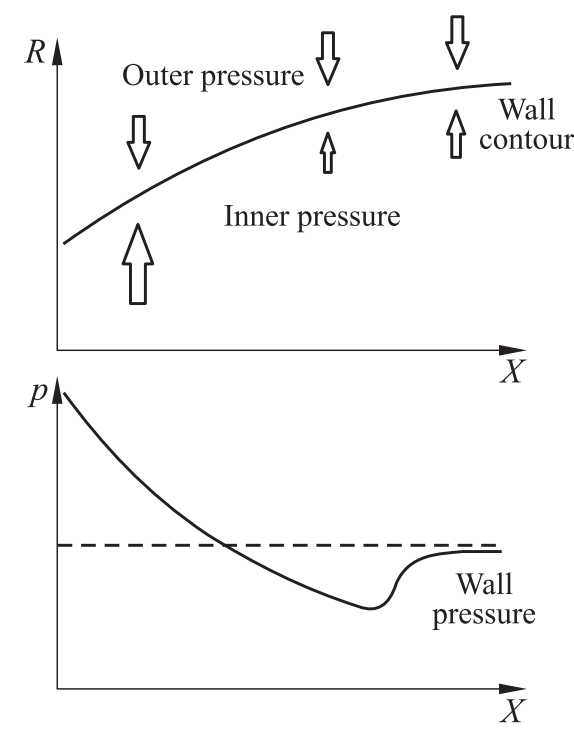

(b)

Figure 1 Wall pressure in perfectly symmetric nozzles, full flowing $(a)$ and separated $(b)$

nozzle is full flowing and the flow attaches to the wall over the entire nozzle inner surface. For low NPRs, the flow is separated, i.e., the flow detaches from the nozzle wall, resulting in a back flow region downstream the separation position where gradual adaptation to ambient pressure occurs (Fig. 1b). The latter flow condition is present during the transient start-up and shutdown of a rocket engine under sea-level conditions, and is, therefore, of special interest.

In case of a full flowing nozzle, an inward bended nozzle wall will increase the recompression characteristics of the wall. As a result, the pressure difference causing the bending force will decrease (Fig. 2a). The bending effect is damped. An outward bended nozzle wall, in the other way, will reduce the recompression characteristics, leading the flow to expand to lower pressure values (Fig. 2b). The pressure difference between inner and outer nozzle wall increases, also damping the bending effect.

A separated nozzle flow behaves differently. An inward bended nozzle wall shifts the flow separation position further downstream and increases, therefore, the lever arm of the bending force (Fig. 2c). This is a self-reinforcing effect. Consequently, an outward bended nozzle wall shifts the flow separation position in upstream direction and the bending is amplified (Fig. $2 d$ ).

Flow disturbances in full flowing nozzles are damped by the flow-structure interaction. In case of separated nozzle flows, the disturbances are amplified 


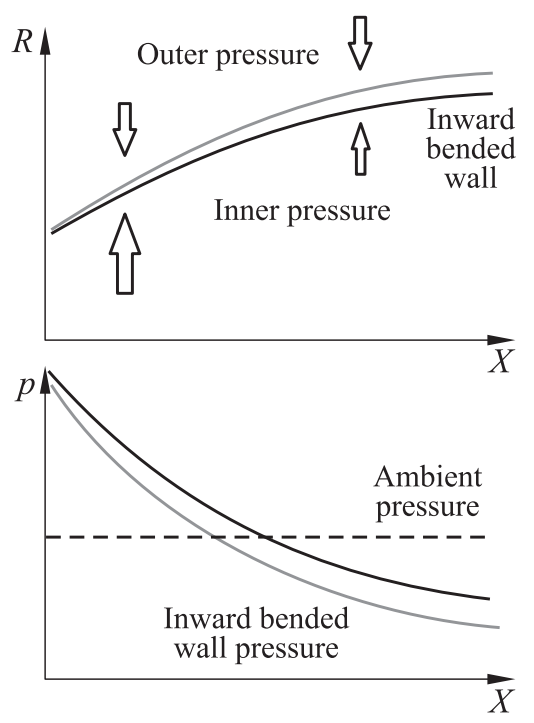

(a)

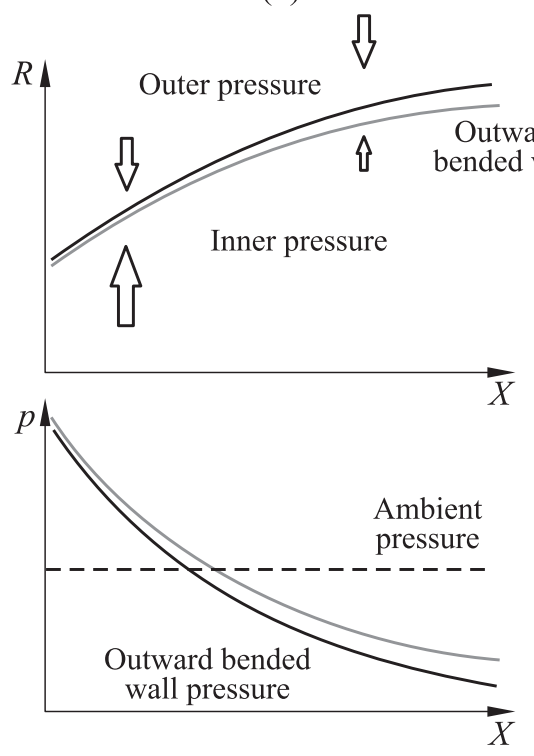

(c)

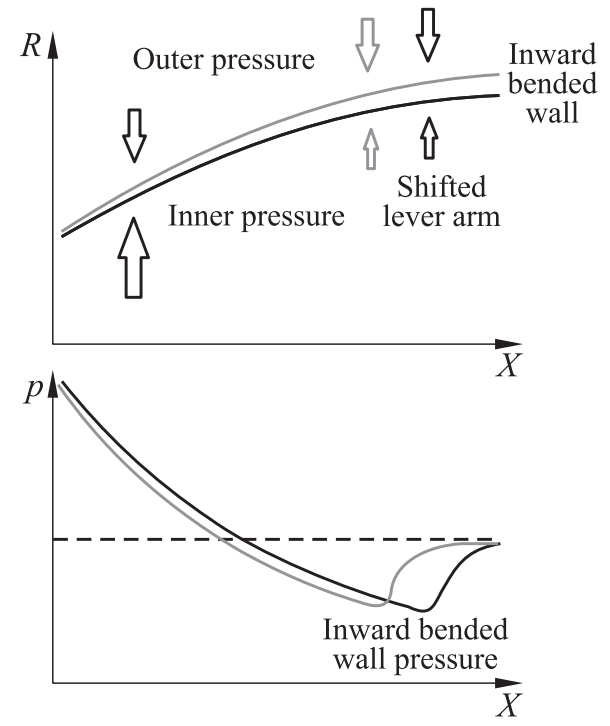

(b)

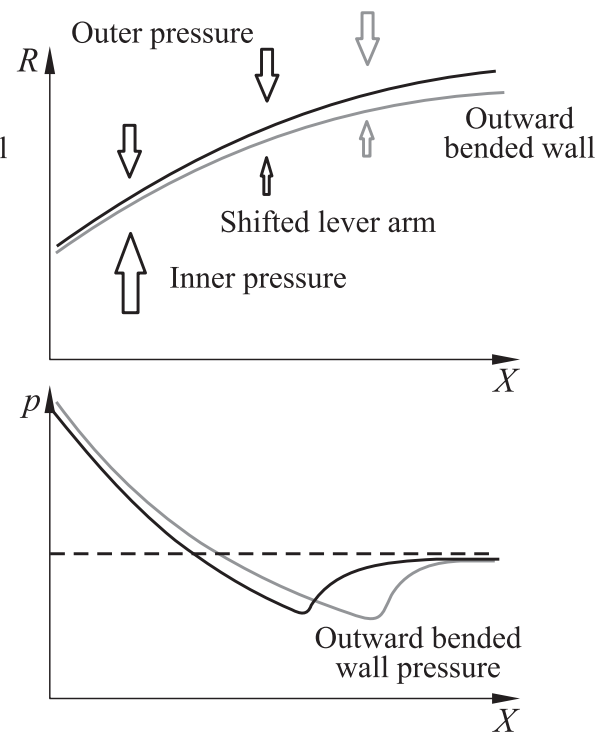

(d)

Figure 2 Wall pressure in deformed nozzles, full flowing (left column) and separated (right column) 


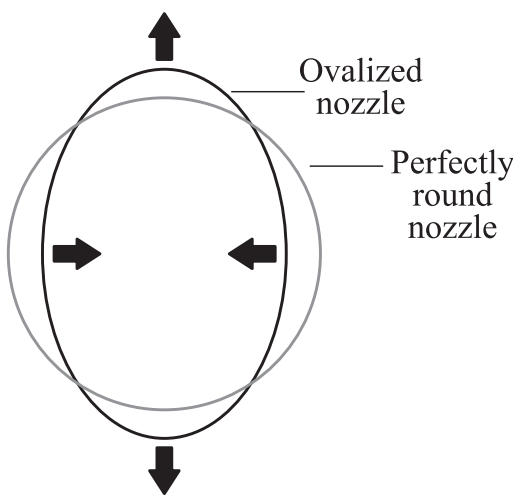

Figure 3 Self-reinforcing nozzle ovalization in case of separated flow

by the flow-structure interaction. These disturbances can be produced either by combustion instabilities or ambient pressure fluctuations. Ambient pressure fluctuations are introduced by the rocket itself during ascent and are the function of the rocket velocity and flight altitude. Under certain conditions, they may excite the bell nozzle eigenfrequency. As a result, the structure responds and the nozzle ovalizes. In case of separated flow, the unsymmetrical separation position generates self-reinforcing pressure forces (Fig. 3). This effect can lead in the last resort to the collapse of the nozzle structural integrity. For this reason, the experimental investigation of flowstructure interaction in ovalized nozzles under separated flow condition is difficult to realize. A join experimental and numerical investigation is presented in this paper. The study has been divided into small logical steps. First, a numerical method is developed and validated for the simulation of separated flow in axisymmetrical nozzle. This method is then applied to permanently ovalized nozzles, studying the flow response to steady structure deformation. The results define the frame and necessary instrumentation for experimental investigation. Once the numerical method is fully validated, it will be implemented to investigate flow-structure interaction in out-of-round nozzles.

The present paper studies different ovalization approaches to assist the selection of future cold flow subscale test specimens. As initial contours truncated, ideal nozzles were chosen to avoid the secondary effect of reattached flow conditions. The work is a cooperation of DLR Lampoldshausen and DLR Braunschweig within the framework of the DLR intern project ProTAU.

\section{LITERATURE STUDY}

Most of the works found in the literature which discuss the flow behavior and structural interaction in out-of-round rocket nozzles are numerical approaches. The experimental study of a flow-structure interaction demands the simultaneous measuring of the flow properties and the structural change, which is more than challenging.

The first example of an experimental study was published by Tuovila and Land in 1968 [1]. The aeroelastic stability of conical rocket nozzles exhausting into a vacuum chamber was studied. For this purpose, a stiff base nozzle 
was prolongated with steel or fiberglass extensions. Whereas the steel nozzle extensions kept stable during test run, the fiberglass nozzles showed ovalization modes with stationary wave forms and high amplitudes. The authors proposed an empirical buckling parameter to predict the stability of conical nozzles.

Recent activities were reported by Brown et al. [2] and Moreaux and Girard [3]. Brown et al. tested a thin walled cold gas TIC (truncated ideal contour) nozzle with strain gauges applied directly downstream the nozzle throat. The wall pressures upstream the flow separation showed fluctuations with frequencies being identical with the nozzle ovalization modes. From the authors point of view, the separation movement is triggered by the ovalization frequency.

Moreaux and Girard [3] studied a TIC nozzle manufactured out of laminated composite material. The nozzle model was tested under full flowing as well as under separated conditions to study the aeroelastic coupling. The authors reported a strong ovalization for NPR $=15$, completed by an additional focal point for NPR $>60$. In tendency, the ovalization frequencies decrease and the damping rises with increasing NPR. The tests ended with the nozzle destruction.

Introduction on separated nozzle flows by Östlund et al. [4] includes, beside flow topology, FSS-RSS (free shock separation-restricted shock separation) transition and related side loads, a calculation of a bended nozzle where the throat is the focal point of the deflection. The comparison of calculations and experimental results showed the awaited trend: increased wall pressures on the inward displaced wall and decreased pressures on the outward bended one.

Xia and Schwane [5] performed a CFD/FEM (computational fluid dynamics / finite element method) cold flow nozzle study related to side loads, induced by bending and ovalization. It shows up that side loads increase with increasing deflection frequency (amplitude kept constant). The study of the flow inside the ovalized nozzle led to surprising results: the flow separates on the inward bended wall further upstream than on the outward bended one. The resulting wall pressure distribution was then transferred to a FEM model and pressure fluctuations were applied on the nozzle inlet condition. As a result, low frequencies damp the ovalization tendency, medium frequencies (around $50 \mathrm{~Hz}$ ) excite the second mode, and higher frequencies (up to $200 \mathrm{~Hz}$ ) increase the deformation.

The paper was followed by a three-dimensional (3D) simulation [6] of the VAC S6 cold flow nozzle. This numerical nozzle model was deformed using a linear excentricity trend. The results reproduce the unexpected separation position shift. The set of papers was completed with another work on ovalization and side loads [7]. The calculation shows an experimentally not observed cap shock pattern within a TIC nozzle. It has to be assessed in combination with its forerunner [5] and gives additional information. Interesting are the included wall pressure calculations of an ovalized Vulcain 2 nozzle. At the outward bended wall, a nearly constant wall pressure profile with a slight negative pressure gradient is given, which might explain the separation downstream shift. The axial 
and radial force distribution as a function of frequency completes the work. The axial force fluctuation is within the range of the determined separation shock movement, the radial amplification lies above the shock motion.

Lüdecke et al. [8] simulated the flow around Ariane 5 at Mach number of 0.8. The complete launcher with its complex structures of lines, struds, and the helium tank was modeled. The results were compared with experimental data obtained from a wind tunnel model. The simulated pressure fluctuations are in good conformance with the experimental results. A strongly asymmetric flow distribution is introduced by the helium tank which leads to nozzle ovalization effects. However, the displacement of the nozzle is, with $0.01 \%$ of the exit diameter, comparably low.

Berggren [9] sets up in cooperation with VAC a Vulcain 2 combustion chamber FEM model to replace the existing one. The work includes a paragraph concerning the resulting ovalization behavior. The main outcome is whether a field of force or an external, sinusoidal distributed pressure load causes the ovalization is irrelevant. In addition, a Vulcain $2+$ (sandwich design) nozzle extension was studied.

A lot of numerical efforts concerning the flow in an deformed J2-X rocket engine, its nozzle, and the resulting side loads were carried out by Wang et al. $[10,11]$. The transient start-up process was calculated for an altitude condition of $30.5 \mathrm{~km}$ with time accurate fluid composition and properties. Four nozzle configurations were studied: the nominal rotationally symmetric configuration, a slightly, a more out-of-round, and a significantly out-of-round nozzle. As J-2X features only one turbine exhaust gas (TEG) inlet duct, a significantly asymmetric film injection distribution is present with a maximum mass flow deviation of $9 \%$.

The transient 3D studies differ in the way the ovalization was applied. At first [10], the ovalization was obtained starting at the injection plate of the thrust chamber with a constant lateral contraction down to the nozzle exit area. For the continuing study [11], only the nozzle extension downstream the TEG injection torus was ovalized but the lateral contraction of the nozzle exit areas was kept constant. In addition, the number of grid cells was doubled, mainly, due to an increase of the radial resolution. The conclusion was as follows: the asymmetric distributed TEG film injection causes a partially reattached flow condition being responsible for the maximum side load. Surprisingly, the significantly out of round nozzle causes the lowest side load increase. The authors propose a flow model where opposite flow effects interact with each other.

\section{CONTOUR DEFORMATION METHODS}

The main objective of the present investigation is to develop a numerical method for the flow prediction in out-of-round rocket nozzles. Nozzle deformation must 
be treated as a flow-structure interaction problem. For a better understanding of the problem, the first step of the investigation was to decouple the problem into a plain aerodynamic aspect in permanently out-of-round nozzles. Therefore, three nozzle contours have been chosen as start models. This study concentrated on ideal nozzle contours. The internal shock free nozzle flow was found favorable for a first approach of the problem.

The contours are designated as TIC-48, TIC-53, and TIC-58 corresponding, respectively, to a design Mach number of 4.8, 5.3, and 5.8. Three deformation parameters were studied: the degree of deformation, its evolution, and its start position along the contour. The maximal deformation amplitude $a_{\mathrm{def}}$ was set at $5 \%, 10 \%$, and $15 \%$ related to the initial contour exit radius $R_{e}$ for the major axis (Table 1, geometries 1 to 3 ). The deformation amplitude was chosen deliberately exagerated (expected amplitudes are around $3 \%$ ), as the main objective of this study is to develop and validate the numerical method and for this reason, the deformations have to be experimentally measurable. Two positions were chosen for the deformation start. The first one was set at the nozzle throat for a deformation applied to the whole nozzle contour, although in reality, the nozzle structure is very stiff in the throat region. The high height-to-width ratio of the cooling channels in this region imposes a thick wall structure, followed with a much thinner structure downstream to the interface point usually located at an area ratio $\epsilon=5$. Therefore, the second approach sets the deformation start for $\epsilon=5$. The evolution from a circular contour to an ovalized contour at the nozzle end can theoretically follow different functions. At this point of the study, not enough information was available on the deformation evolution along the nozzle contour. Two approaches were arbitrary chosen for validation purposes: a linear and a parabolic axial evolution of the deformation (geometries 5 and 4).

The parametric study was divided into seven numerical test cases, which are summarized in Table 1. Figure 4 illustrates one of the geometries before and after deformation.

Table 1 Parameters of deformation for the seven geometries

\begin{tabular}{ccccl}
\hline Geometry & Reference nozzle & $\begin{array}{c}\text { Amplitude } \\
\left(a_{\mathrm{def}} / R_{e}\right)\end{array}$ & $\begin{array}{c}\text { Start of } \\
\text { ovalization, } \epsilon\end{array}$ & $\begin{array}{c}\text { Deformation } \\
\text { function }\end{array}$ \\
\hline 1 & TIC-48 & $5 \%$ & 5 & Polynomial \\
2 & TIC-48 & $10 \%$ & 5 & Polynomial \\
3 & TIC-48 & $15 \%$ & 5 & Polynomial \\
4 & TIC-53 & $10 \%$ & 5 & Polynomial \\
5 & TIC-53 & $10 \%$ & 5 & Linear \\
6 & TIC-53 & $10 \%$ & 1 & Polynomial \\
7 & TIC-58 & $10 \%$ & 5 & Polynomial \\
\hline
\end{tabular}




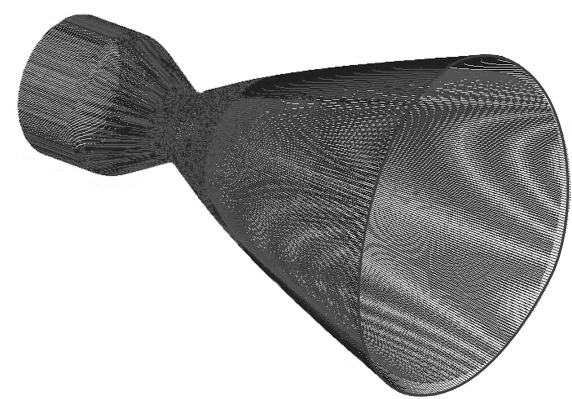

Figure 4 View of the initial contour TIC-58 with the deformation geometry 7

\section{NUMERICAL METHOD}

To assess the defined ovalized geometries and identify promising candidates with regard to a measureable influence of the deformation, CFD simulations of all geometries have been carried out for various NPR values.

All numerical simulations were performed with the DLR finite-volume solver TAU. For the computations, stationary Reynolds-averaged Navier-Stokes equations were solved using the original Spalart Allmaras turbulence model to close the turbulent equations. This one-equation turbulence model has been proven to show the best results for separated nozzle flow simulations [12]. For the fluid model, nitrogen was defined as perfect gas. All simulations have been carried out on 3D hybrid grids of a quarter section of the nozzle (90 degree opening angle) with adiabatic walls. Further investigations will show if the symmetry planes have a notable effect on the shape and position of the separation line and the shock system.

\section{EXPERIMENTAL METHOD}

Three test specimen of the undeformed nozzles were manufactured and tested; the measurements were used for the validation of numerical data. The nozzle models feature different design Mach numbers (Table 2), hence opening wall angle. The ideal contours were truncated to ensure full flowing condition for $\mathrm{NPR}=50$.

The tests were conducted at the test facility P6.2 at DLR in Lampoldshausen. The bench is designed for the cold flow investigation of subscale nozzles under ambient or high altitude conditions, thanks to its two test positions: a horizontal rig and an altitude chamber. The working medium is dry nitrogen. For the present study, the tests were conducted under ambient conditions, on the 
Table 2 Geometrical parameters of the nozzle models

\begin{tabular}{ccccc}
\hline $\begin{array}{c}\text { Nozzle } \\
\text { model }\end{array}$ & $\begin{array}{c}\text { Design } \\
\text { Mach number }\end{array}$ & $\begin{array}{c}\text { Throat } \\
\text { radius } R^{*}, \text { mm }\end{array}$ & $\begin{array}{c}\text { Area } \\
\text { ratio } \epsilon\end{array}$ & $\begin{array}{c}\text { Wall exit } \\
\text { angle }\end{array}$ \\
\hline TIC-48 & 4.8 & 10 & 16.9 & $5.0^{\circ}$ \\
TIC-53 & 5.3 & 10 & 18.5 & $8.5^{\circ}$ \\
TIC-58 & 5.8 & 10 & 19.3 & $11.3^{\circ}$ \\
\hline
\end{tabular}

horizontal rig. The feeding pressure can be regulated between 1 and 55 bar, following pressure ramps with various gradients.

The nozzle models were equipped with wall pressure ports placed regularly along the contour in 4-millimeter interval. The 0.5-millimeter orifices drilled in the wall connected the flow with the pressure transducers through a combination of small pipes glued in the wall and Teflon tubes. Piezoresistive 'Kulite' transducers, model XT-154-190M, measured the wall pressure with a scan rate of $1 \mathrm{kHz}$. The signals were filtered with a 160-hertz low pass filter.

\section{VALIDATION}

Figure 5 shows the comparison of the wall pressure distribution computed by the CFD solver and the one measured in the experiment for the TIC-48 and TIC-53 undeformed geometries.

The simulation shows good results concerning the separation position: for NPR $>30$, the distance between calculated and measured separation positions is within the sensor interval. Although the overall agreement with the experimental data seems to be satisfying, general deviations can be observed. The pressure gradient downstream the separation position is calculated much steeper than it was measured during the experiment. Furthermore, the computational model seems to tend to underpredict the static pressure in the recirculation area. The combination of these two effects, which can be found in earlier studies $[12,13]$, leads to a deviation of the calculated separation position which depends on the size of the recirculation area and, therefore, on the nozzle pressure ratio. Detailed investigations will have to be carried out in future simulations on how this dependency is influenced by the simulation and numerical parameters, e.g., the geometry and the used turbulence model, since for the deformed geometries, the separation position varies with the intersection angle along the nozzle wall.

Figure 6 is a comparison of the wall pressure distribution in the TIC-48 nozzle calculated with CFD and the method of characteristics (MOC) for an axisymetrically deformed contour. The contour related to the major and minor axes of geometry 2 were considered in this case as two-dimensional (2D) nozzle contours. Both methods show a similar wall pressure distribution. This means that the flow separation position along the main deformation direction $\left(0^{\circ}\right.$, major 


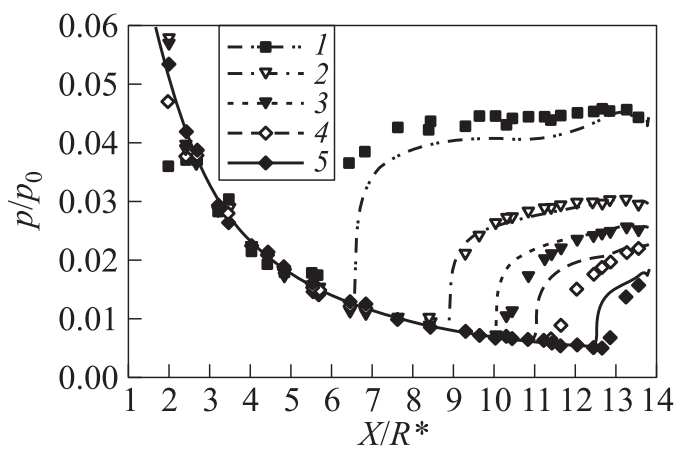

(a)

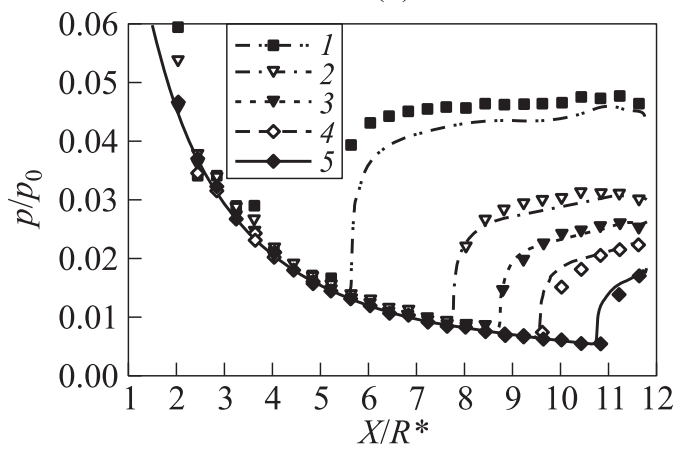

(b)

Figure 5 Wall pressure distribution (experiment and CFD) in the undeformed TIC$48(a)$ and TIC-53 (b) nozzles for various NPR values: $1-20 ; 2-30 ; 3-35 ; 4-$ 40 ; and $5-50$

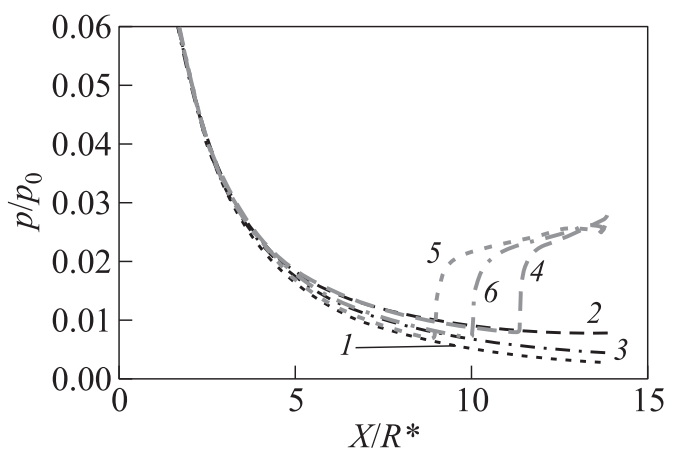

Figure 6 Wall pressure distribution calculated with MOC (vacuum profiles) and CFD (for NPR = 35): 1 - MOC, major axis; 2 - MOC, minor axis; 3 - vacuum profile, undeformed contour; $4-\mathrm{CFD}$, minor axis; $5-\mathrm{CFD}$, major axis; and $6-$ $\mathrm{CFD}$, undeformed contour 
axis and $90^{\circ}$, minor axis) can be reasonably estimated in the first approximation using 2D MOC calculations.

\section{EFFECTS OF OVALIZATION}

Figure 7 illustrates the position of the separation line within the deformed geometry 2 for NPR $=35$. On the major axis, the outward bended wall shifts the flow separation upstream while on the minor axis, the inward bended nozzle wall shifts the separation downstream.

To measure and visualize the effects of the deformation parameters on the flow characteristics, the separation line and the Mach disk were automatically extracted from the flow field data and appropriate functions were fitted to their geometries.

For the separation detection, the correspondent function of the postprocessing software tecplot 360 was used. This feature is based on the MIT FX library [14]. A distorted sine function was fitted to the data:

$$
X=a_{\mathrm{sep}} \sin \left(2 \varphi+\Delta \varphi+c \sin \left(2\left(\varphi-\frac{\pi}{2}\right)\right)\right)+\bar{X}_{\mathrm{sep}} .
$$

Hereby, the variable $a_{\text {sep }}$ indicates the amplitude of the separation position variation in axial direction.

The Mach disk was detected by analyzing the local maxima in the pressure gradient magnitude. In the cross section, an hyperbolic paraboloid was fitted to the data, the radial section is again described by a sine function. The following equations were used to define the saddle shaped Mach disk:

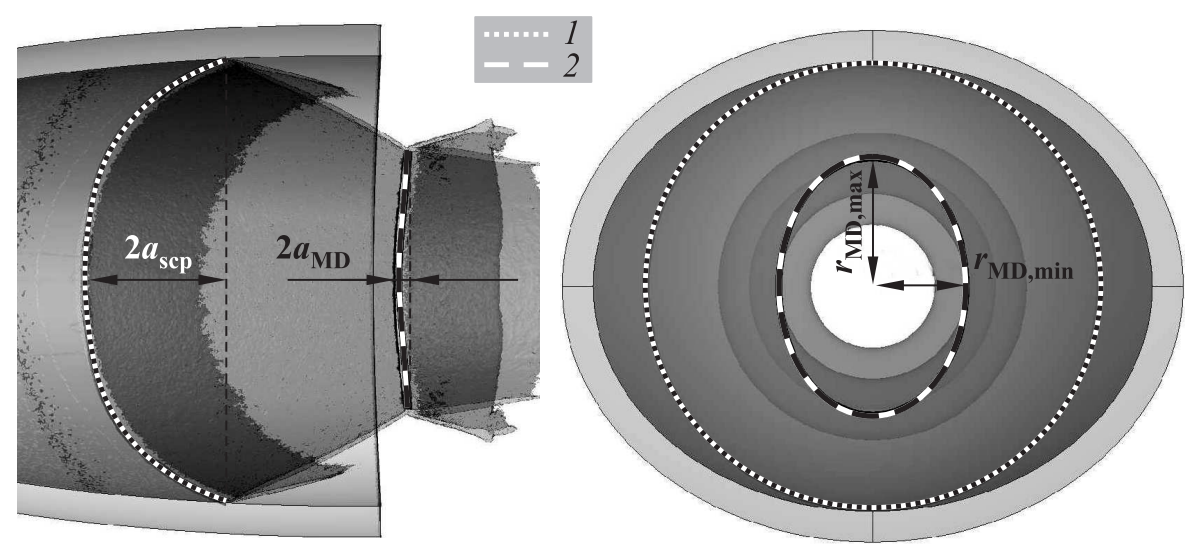

Figure 7 Mach disk (1) and separation line (2) for geometry 2 and NPR $=35$ 


$$
\begin{aligned}
X_{\mathrm{MD}} & =a_{\mathrm{MD}} \sin (2 \varphi+\Delta \varphi)+\bar{X}_{\mathrm{MD}} ; \\
1 & =\left(\frac{Z_{\mathrm{MD}}}{r_{\mathrm{MD}, \text { max }}}\right)^{2}+\left(\frac{Y_{\mathrm{MD}}}{r_{\mathrm{MD}, \text { min }}}\right)^{2}
\end{aligned}
$$

Here, $a_{\mathrm{MD}}$ stands for the amplitude of the axial variation of the Mach saddle border, corresponding to the triple point in $2 \mathrm{D}$ view. All fits were calculated using the least square method. Figure 7 shows a typical flow pattern and the fitted functions.

The results of the simulations basically show the tendencies expected by a $2 \mathrm{D}$ simplification on the intersections of an ovalized nozzle. Figure $8 a$ shows the amplitude of the separation-fitted sine function plotted over the deformation amplitude. It can be seen that there seems to be a linear correlation between the two plotted amplitudes. Furthermore, the NPR has also a significant influence on the sine amplitude (Fig. 9). With rising total pressure, the separation line deformation increases until the most downstream separation point reaches the nozzle end. The influence of the NPR increases for shorter nozzles with a higher wall exit angle. The maximal sine amplitude lies at a NPR of 35 or 40 depending on the geometry. It should be noted that the above mentioned underprediction of the pressure in the recirculation area might affect this correlation.

A higher deformation of the nozzle also emphasizes the deformation of the Mach disk, both in radial and axial directions (see Fig. $8 b$ ). This again is in good agreement with a $2 \mathrm{D}$ consideration and the mentioned effects on the separation. The geometry can, therefore, be designed so that the separation line, as well as the shock system, show a significant variation.

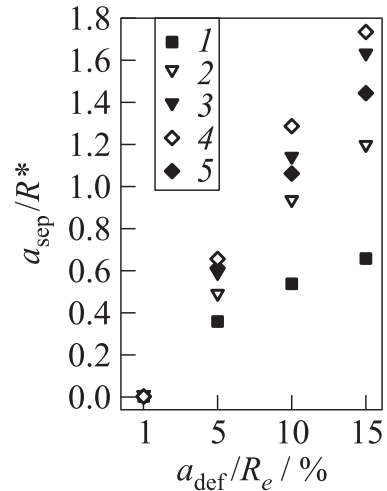

(a)

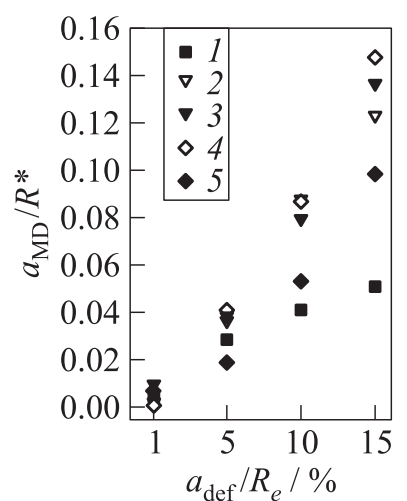

(b)

Figure 8 Fitted separation $(a)$ and Mack disk sine amplitudes over deformation amplitude: $1-\mathrm{NPR}=20 ; 2-30 ; 3-35 ; 4-40 ;$ and $5-\mathrm{NPR}=50$ 


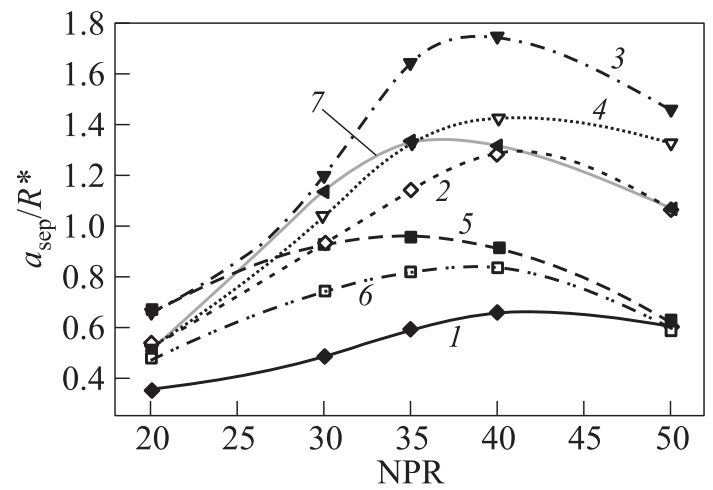

Figure 9 Separation fit amplitude over NPR: $1-$ geometry $1 ; 2-2 ; 3-3 ; 4-$ $4 ; 5-5 ; 6-6$; and $7-$ geometry 7

The simulations have shown (see Fig. $8 a$ ) an amplitude of the separation line deformation in a measurable range for an ovalization $a_{\mathrm{def}} / R^{*}$ over $10 \%$. The choice of the deformation function (geometries 4 and 5) also demonstrated a flow with separation variation in a range of a few millimeters (see Fig. 9), hence, a measurable effect in the experiment.

\section{CONCLUDING REMARKS}

The first joint investigation, numerical and experimental, has been conducted to study the effect of rocket nozzle ovalization on flow behavior. Various deformations have been applied to three TIC nozzle contours. The flow separation position were calculated with the TAU code for various NPR in the initial and the deformed nozzles using 3D CFD simulations. The results of the simulation were validated for the undeformed nozzles with cold flow experimental data. The contour deformations have shown a measurable effect on the flow topology. The next step is the validation of the data through experimental investigation of some of the permanently deformed geometries, before going into the flow-structure interaction.

\section{ACKNOWLEDGMENTS}

Financial support has been provided for the experiment by the German Research Foundation (Deutsche Forschungsgemeinschaft - DFG) in the framework of the Sonderforschungsbereich Transregio 40. The authors would like to thank Dr. Sebastian Karl (DLR Göttingen) for his help on mesh generation, modification of the TAU code, and expertise on its application for nozzle simulations. 


\section{REFERENCES}

1. Tuovila, W. J., and N. S. Land. 1968. Experimental study of aeroelastic instability of overexpanded rocket nozzle extensions. Langley Research Center. NASA-TN-D4471.

2. Brown, A. M., J. Ruf, D. Reed, M. D'Agostino, and R. Keanini. 2002. Characterization of side load phenomena using measurement of fluid/structure interaction. AIAA Paper No. 2002-3999.

3. Moreaux, N., and S. Girard. 2003. Experimental assessment of aeroelastic coupling in a rocket engine nozzle. IFASD 2003. Amsterdam, The Netherlands. ONERA TP 2003-80.

4. Östlund, J., T. Damgaard, and M. Frey. 2001. Side-load phenomena in highly overexpanded rocket nozzles. AIAA Paper No. 2001-3684.

5. Xia, Y., and R. Schwane. 2004. Investigation of the aerodynamic influence of a non-axisymmetric flow on side load generation in over-expanded rocket nozzles. Deutscher Luft- und Raumfahrtkongress. DGLR-JT2004-274.

6. Schwane, R., and Y. Xia. 2004. On the dynamics of shock waves in over-expanded rocket nozzles. AIAA Paper No. 2004-1128.

7. Schwane, R., and Y. Xia. 2005. Time-accurate CFD predictions and data validation for side load generation by flow-structure coupling in over-expanded rocket nozzles. J. Math. Model. Algorithm 4(1):53-65.

8. Lüdeke, H., J. B. Calvo, and A. Filimon. 2006. Fluid structure interaction at the Ariane-5 nozzle section by advanced turbulence models. European Conference on Computational Fluid Dynamics (ECCOMAS CFD). TU Delft, The Netherlands.

9. Berggren, J. 2007. Improved stability analysis of rocket engine nozzles. Luleå University of Technology. Master's Thesis.

10. Wang, T.-S., J. Lin, J. Ruf, and M. Guidos. 2011. Transient three-dimensional sideload analysis of out-of-round film-cooled nozzles. J. Propul. Power 27(4):899-907.

11. Wang, T.-S., J. Lin, and M. Guidos. 2012. Transient side load analysis of out-ofround film-cooled nozzle extensions. AIAA Paper No. 2012-3968.

12. Stark, R., and G. Hagemann. 2007. Current status of numerical flow prediction for separated nozzle flows. 2nd European Conference for Aerospace Science. Brussels, Belgium.

13. Wagner, B., S. Karl, and K. Hannemann. 2006. Test case 1a: Short nozzle under separated flow condition and numerical investigation with the DLR Tau-code. FSCD-ATAC Workshop. Nordwijk, The Netherlands.

14. FX - Fluid feature eXtraction tool-kit. Available at: http://raphael.mit.edu/fx (accessed July 7, 2014). 